\title{
Thermodynamics of charged, rotating, and accelerating black holes
}

\author{
Andrés Anabalón, ${ }^{a}$ Finnian Gray, ${ }^{b, c}$ Ruth Gregory, ${ }^{d, b}$ David Kubiznák ${ }^{b, c}$ \\ and Robert B. Mann ${ }^{c, b}$ \\ ${ }^{a}$ Departamento de Ciencias, Facultad de Artes Liberales, Universidad Adolfo Ibáñez, \\ Avenida Padre Hurtado 750, Viña del Mar 2520000, Chile \\ ${ }^{b}$ Perimeter Institute, \\ 31 Caroline St., Waterloo, Ontario, N2L 2Y5, Canada \\ ${ }^{c}$ Department of Physics and Astronomy, University of Waterloo, \\ Waterloo, Ontario, N2L 3G1, Canada, \\ ${ }^{d}$ Centre for Particle Theory, Durham University, \\ South Road, Durham, DH1 3LE, U.K. \\ E-mail: andres.anabalon@uai.cl, fgray@perimeterinstitute.ca, \\ R.A.W.Gregory@durham.ac.uk, dkubiznak@perimeterinstitute.ca, \\ rbmann@uwaterloo.ca
}

ABSTRACT: We show how to obtain a consistent thermodynamic description of accelerating asymptotically AdS black holes, extending our previous results by including charge and rotation. We find that the key ingredient of consistent thermodynamics is to ensure that the system is not over-constrained by including the possibility of varying the 'string' tensions that are responsible for the acceleration of the black hole, yielding a first law of full cohomogeneity. The first law assumes the standard form, with the entropy given by one quarter of the horizon area and other quantities identified by standard methods. In particular we compute the mass in two independent ways: through a Euclidean action calculation and by the method of conformal completion. The ambiguity in the choice of the normalization of the timelike Killing vector can be fixed by explicit coordinate transformation (in the case of rotation) to the standard AdS form or by holographic methods (in the case of charge). This resolves a long-standing problem of formulating the thermodynamics of accelerating black holes, opening the way to detailed studies of their phase behaviour.

KeYwords: Black Holes, AdS-CFT Correspondence

ArXIV EPRINT: 1811.04936 


\section{Contents}

1 Introduction 1

2 Generalized C-metric 2

3 Slowly accelerating black hole thermodynamics 4

4 On thermodynamics of the asymptotically flat C-metric $\quad 8$

$\begin{array}{lll}5 & \text { Discussion and summary } & 10\end{array}$

$\begin{array}{ll}\text { A Holographic stress tensor } & 11\end{array}$

\section{Introduction}

Black holes provide us with an invaluable and unique tool for probing the relationship between quantum physics and the gravitational force. From the early discoveries that black hole area and surface gravity respectively behave as thermodynamic entropy $[1,2]$ and temperature [3], a rich and varied range of thermodynamic behaviour of these objects has been uncovered [4], providing new insight into the underlying microscopic degrees of freedom that may be associated with quantum gravity.

Amongst the panoply of black hole solutions, accelerating black holes have been somewhat less well understood. Described by the so-called $C$-metric [5-8], they have a conical singularity along one, or both, polar axes. Replacing this with a stress-energy tensor of a finite width cosmic string [9], or magnetic flux tube [10], gives a concrete interpretation of the force that accelerates the black holes. Although they have been used in a variety of settings to demonstrate that the pair creation rate of black holes is proportional to their entropy [9-15], their thermodynamics has remained perplexing, particularly when charge and rotation are included. Conflicting results have appeared in the literature [16-20] concerning the relationship between the conserved mass and its associated thermodynamic quantity, the role of conical deficits in the first law, and the relationship between the action and the free energy. Recently these inconsistencies were resolved for the non-rotating uncharged accelerating black hole in [21], by exploring the holographic properties of the accelerating black hole in AdS with no acceleration horizon, the slowly accelerating black hole [22]. Since there is only one horizon, the system has a unique temperature and thermodynamics is straightforward to define.

Here we consider the full thermodynamics of slowly accelerating black holes, including charge and rotation, and find all relevant thermodynamic variables showing that both the extended first law [17]

$$
\delta M=T \delta S+\Phi \delta Q+\Omega \delta J-\lambda_{+} \delta \mu_{+}-\lambda_{-} \delta \mu_{-}+V \delta P,
$$


and the Smarr relation [23]

$$
M=2(T S+\Omega J-P V)+\Phi Q,
$$

hold in all cases. We emphasize that both the string tensions $\mu_{ \pm}$, as well as the cosmological constant, $\Lambda=-8 \pi P$, are allowed to vary, and we derive the associated thermodynamic lengths, $\lambda_{ \pm}$, and the thermodynamic volume, $V$, as their respective conjugate quantities. The scaling properties of $\mu_{ \pm}$prevent their appearance in the Smarr relation (1.2). As we shall see, the inclusion of the tension terms is absolutely crucial for the laws of thermodynamics to take their natural form.

\section{Generalized C-metric}

We begin by introducing the generalized AdS C-metric solution as derived from the Plebański-Demiański metric [6] in Boyer-Lindquist type coordinates [8] to include rotation, charge, cosmological constant $\Lambda=-3 / \ell^{2}$, and the corresponding gauge potential

$$
\begin{aligned}
d s^{2}=\frac{1}{H^{2}}\{ & -\frac{f(r)}{\Sigma}\left[\frac{d t}{\alpha}-a \sin ^{2} \theta \frac{d \varphi}{K}\right]^{2}+\frac{\Sigma}{f(r)} d r^{2} \\
& \left.+\frac{\Sigma r^{2}}{h(\theta)} d \theta^{2}+\frac{h(\theta) \sin ^{2} \theta}{\Sigma r^{2}}\left[\frac{a d t}{\alpha}-\left(r^{2}+a^{2}\right) \frac{d \varphi}{K}\right]^{2}\right\} \\
F=d B, \quad & B=-\frac{e}{\Sigma r}\left[\frac{d t}{\alpha}-a \sin ^{2} \theta \frac{d \varphi}{K}\right]+\Phi_{t} d t,
\end{aligned}
$$

where we choose

$$
\Phi_{t}=\frac{e r_{+}}{\left(a^{2}+r_{+}^{2}\right) \alpha}
$$

so that the gauge potential, defined by $-\xi \cdot B$, where $\xi$ is the generator of the horizon, $\xi=\partial_{t}+\Omega_{H} \partial_{\varphi}$ (where $\Omega_{H}$ is defined in (3.1) below), vanishes at the horizon. The metric functions are given by

$$
\begin{gathered}
f(r)=\left(1-A^{2} r^{2}\right)\left[1-\frac{2 m}{r}+\frac{a^{2}+e^{2}}{r^{2}}\right]+\frac{r^{2}+a^{2}}{\ell^{2}}, \\
h(\theta)=1+2 m A \cos \theta+\left[A^{2}\left(a^{2}+e^{2}\right)-\frac{a^{2}}{\ell^{2}}\right] \cos ^{2} \theta, \\
\Sigma=1+\frac{a^{2}}{r^{2}} \cos ^{2} \theta, \quad H=1+A r \cos \theta .
\end{gathered}
$$

As in past work [16-18], we focus on the slowly accelerating black hole that has no acceleration horizon, i.e. the only relevant zero of $f$ being the black hole horizon. The conformal factor $H$ then sets the location of the boundary at $r_{\mathrm{bd}}=-1 / A \cos \theta$.

It is worth remarking on the various parameters appearing in the metric: $m, a, e$ and $\ell$ are the usual parameters representing the mass, rotation, charge of the black hole and the cosmological constant respectively. Loosely speaking, $A$ encodes the acceleration of the black hole, and $K$ the conical deficit of the spacetime. Often, the $K$ factor is absorbed in 
the azimuthal coordinate, which in turn would have an arbitrary periodicity, usually fixed by a regularity condition at one of the poles. We include the explicit factor of $K$ so that the periodicity of $\varphi$ is fixed at $2 \pi$. The conical deficit at each axis is then found by checking the behaviour of the $\theta-\varphi$ part of the metric near $\theta=\theta_{ \pm}=0, \pi$ respectively:

$$
d s_{\theta, \varphi}^{2} \propto d \theta^{2}+h^{2}(\theta) \sin ^{2} \theta \frac{d \varphi^{2}}{K^{2}} \sim d \vartheta^{2}+(\Xi \pm 2 m A)^{2} \vartheta^{2} d \varphi^{2},
$$

where $\vartheta_{ \pm}= \pm\left(\theta-\theta_{ \pm}\right)$gives a local radial coordinate near each axis, and

$$
\Xi=1-\frac{a^{2}}{\ell^{2}}+A^{2}\left(e^{2}+a^{2}\right)
$$

The definition of a conical singularity is that the circumference of a circle, $\mathcal{C}=\Delta \varphi \sqrt{g_{\varphi \varphi}}$, is not equal to $2 \pi$ times its proper radius, $\mathcal{R}=\vartheta \sqrt{g_{\theta \theta}}$. The deficit angle is this discrepancy: $\delta=2 \pi-\mathcal{C} / \mathcal{R}$. This is the reason for the parameter $K$ : by fixing the periodicity of $\varphi$ as $\Delta \varphi=2 \pi$, the deficits at each axis are now given explicitly in terms of the parameters appearing in the metric. Since a deficit angle is typically interpreted as due to the presence of a cosmic string, one deduces that the tensions of the strings along each axis, $\mu_{ \pm}=\delta_{ \pm} / 8 \pi$, are:

$$
\mu_{ \pm}=\frac{1}{4}\left[1-\frac{\Xi \pm 2 m A}{K}\right] .
$$

We therefore see that acceleration is due to a mismatch of conical deficits from the North to South pole: $\mu_{-}-\mu_{+}=m A / K$, whereas $K$ tracks an overall deficit in the spacetime: $\bar{\mu}=\left(\mu_{+}+\mu_{-}\right) / 2=\frac{1}{4}(1-\Xi / K)$.

Thus far we have related all parameters in the metric to physical charges. We emphasize that $\alpha$, the parameter rescaling $t$, is not a new parameter: since $t$ is a noncompact coordinate it is not a physical parameter. Rather it is a choice of gauge, anticipating that the $t$ coordinate natural to the $(t, r)$ section of a black hole metric is not, in fact, the correctly scaled coordinate when viewed from the perspective of an observer at infinity (for the non accelerating Kerr-AdS, the presence of this rescaling was first noted in [24].) We insert $\alpha$ at this stage to anticipate this rescaling - see (3.2) below.

To summarize, the general solution above is described by six independent parameters $\{m, a, e, \ell, K, A\}$, which map to six physical charges $\left\{M, J, Q, P, \mu_{+}, \mu_{-}\right\}$, that all appear on the r.h.s. of the First Law (1.1). As a consequence the first law has "full cohomogeneity".

Finally, in order for the metric (2.1) to be well defined, and correspond physically to a slowly accelerating black hole in the bulk, we must restrict the range of parameters as follows:

1. For the obvious interpretation of the $\theta$-coordinate, we must have $h(\theta)>0$ on $[0, \pi]$, which implies

$$
m A<\left\{\begin{array}{l}
\frac{1}{2} \Xi \text { for } \quad \Xi \in(0,2], \\
\sqrt{\Xi-1} \text { for } \Xi>2 .
\end{array}\right.
$$

(Other ranges of $\theta$ where $h$ is positive yield, for example, hyperbolic black holes [25].) 
2. Since the range of $r$ is constrained by the conformal factor, we have ${ }^{1} A \cos \theta \leq$ $1 / r \leq 1 / r_{+}$, and to satisfy the requirement of slow acceleration, we must require that $f(-1 / A \cos \theta)$ has no roots.

3. Finally, we must make sure that the spacetime admits a black hole in the bulk, that is $f(r)$ has to have at least one root in the range $r \in(0,1 / A)$.

Once we establish that we are within these allowed parameter ranges, our solution describes a single accelerating black hole that is suspended by strings at a constant radius in asymptotically AdS space. We refer the reader to reference [37] for the (rather non-trivial and $\theta$-dependent) Penrose diagram of these spacetimes.

\section{Slowly accelerating black hole thermodynamics}

We begin by stating the thermodynamic charges and potentials for the accelerating black hole:

$$
\begin{aligned}
M & =\frac{m\left(\Xi+a^{2} / \ell^{2}\right)\left(1-A^{2} \ell^{2} \Xi\right)}{K \Xi \alpha\left(1+a^{2} A^{2}\right)}, \\
T & =\frac{f_{+}^{\prime} r_{+}^{2}}{4 \pi \alpha\left(r_{+}^{2}+a^{2}\right)}, \quad S=\frac{\pi\left(r_{+}^{2}+a^{2}\right)}{K\left(1-A^{2} r_{+}^{2}\right)}, \\
Q & =\frac{e}{K}, \quad \Phi=\Phi_{t}=\frac{e r_{+}}{\left(r_{+}^{2}+a^{2}\right) \alpha}, \\
J & =\frac{m a}{K^{2}}, \quad \Omega=\Omega_{H}-\Omega_{\infty}=\left(\frac{K a}{\alpha\left(r_{+}^{2}+a^{2}\right)}\right)-\left(-\frac{a K\left(1-A^{2} \ell^{2} \Xi\right)}{\ell^{2} \Xi \alpha\left(1+a^{2} A^{2}\right)}\right), \\
P & =\frac{3}{8 \pi \ell^{2}}, \quad V=\frac{4 \pi}{3 K \alpha}\left[\frac{r_{+}\left(r_{+}^{2}+a^{2}\right)}{\left(1-A^{2} r_{+}^{2}\right)^{2}}+\frac{m\left[a^{2}\left(1-A^{2} \ell^{2} \Xi\right)+A^{2} \ell^{4} \Xi\left(\Xi+a^{2} / \ell^{2}\right)\right]}{\left(1+a^{2} A^{2}\right) \Xi}\right] \\
\lambda_{ \pm} & =\frac{r_{+}}{\alpha\left(1 \pm A r_{+}\right)}-\frac{m}{\alpha} \frac{\left[\Xi+a^{2} / \ell^{2}+\frac{a^{2}}{\ell^{2}}\left(1-A^{2} \ell^{2} \Xi\right)\right]}{\left(1+a^{2} A^{2}\right) \Xi^{2}} \mp \frac{A \ell^{2}\left(\Xi+a^{2} / \ell^{2}\right)}{\alpha\left(1+a^{2} A^{2}\right)},
\end{aligned}
$$

which, together with the tensions $\mu_{ \pm}$defined in (2.7), satisfy both the first law (1.1) and the Smarr relation (1.2), provided we set

$$
\alpha=\frac{\sqrt{\left(\Xi+a^{2} / \ell^{2}\right)\left(1-A^{2} \ell^{2} \Xi\right)}}{1+a^{2} A^{2}}
$$

Let us now turn towards the derivation of these quantities.

$\boldsymbol{T}$ and $\boldsymbol{S}$. The most straightforward of the above parameters is the entropy, given by one quarter of the horizon area [26],

$$
S=\frac{\text { Area }}{4}=\left.\frac{r^{2}+a^{2}}{4 K} \int \frac{\sin \theta}{H^{2}} d \theta d \varphi\right|_{r=r_{+}}=\frac{\pi\left(r_{+}^{2}+a^{2}\right)}{K\left(1-A^{2} r_{+}^{2}\right)}
$$

\footnotetext{
${ }^{1}$ Note that when $\cos \theta<0,1 / r$ crosses the origin and so the boundary is situated 'beyond infinity'.
} 
Next, the Hawking temperature of the black hole, $T$, can be obtained as usual by regularity of the Euclidean section ${ }^{2}$

$$
d s_{\tau, r}^{2} \propto \frac{r^{2} \Sigma}{r^{2}+a^{2}}\left[\frac{r^{2} f}{\left(r^{2}+a^{2}\right)} \frac{d \tau^{2}}{\alpha^{2}}+\frac{d r^{2}}{r^{2} f /\left(r^{2}+a^{2}\right)}\right] \Rightarrow T=\frac{f^{\prime} r_{+}^{2}}{4 \pi \alpha\left(r_{+}^{2}+a^{2}\right)} .
$$

$\boldsymbol{Q}$ and $\boldsymbol{\Phi}$. The electric and magnetic charges of the black hole, $Q$ and $Q_{m}$, can be found by the standard Gauss' law integral

$$
Q=\frac{1}{4 \pi} \int * F=\frac{e}{K} \quad Q_{m}=\frac{1}{4 \pi} \int F=0 .
$$

The conjugate electrostatic potential, $\Phi$, is a little more interesting. The usual procedure is to define the bulk potential $B$ so that the corresponding electrostatic potential $-\xi \cdot B$ vanishes on the horizon of a black hole, and its value at infinity then gives the thermodynamic potential. Here, however, we have the problem that the potential and electric field are not constant at the boundary due the acceleration giving a nontrivial $\theta$-dependence, so we cannot simply read off the potential there. Instead, as already noted in (2.2) and (2.3), we set the electrostatic potential $-\xi \cdot B$ to be zero on the horizon, and define the thermodynamic potential using the Hawking-Ross prescription [27]

$$
\Phi=\frac{1}{4 \pi Q \beta} \int_{\partial M} \sqrt{h} n_{a} F^{a b} B_{b},
$$

where $\beta=1 / T$ is the inverse periodicity in Euclidean time, and $n^{a}$ is the outward pointing unit normal to $\partial M \equiv\{H=0\}$. This computation simply gives $\Phi=\Phi_{t}$, as in (2.3).

$\boldsymbol{J}$ and $\boldsymbol{\Omega}$. The angular momentum $J$ can be computed (for example) by using the conformal method $[28,29]$. The idea is to perform a conformal transformation on the metric, $\bar{g}_{\mu \nu}=\bar{H}^{2} g_{\mu \nu}$, to remove the divergence near the boundary, then to integrate a conserved current associated with the Killing vector $\xi$, to get the corresponding conserved charge:

$$
\mathrm{Q}(\xi)=\frac{\ell}{8 \pi} \lim _{\bar{H} \rightarrow 0} \oint \frac{\ell^{2}}{\bar{H}} N^{\alpha} N^{\beta} \bar{C}^{\nu}{ }_{\alpha \mu \beta} \xi_{\nu} d \bar{S}^{\mu},
$$

where $\bar{C}_{\alpha \nu \beta}^{\mu}$ is the Weyl tensor of the conformal metric and

$$
d \bar{S}_{\mu}=\delta_{\mu}^{t} \frac{\ell^{2}\left(1+a^{2} A^{2} \cos ^{4} \theta\right)}{\alpha K} d(\cos \theta) d \varphi
$$

is the spacelike surface element tangent to $\bar{H}=0$, with the normal to the boundary $N_{\mu}=$ $\partial_{\mu} \bar{H}$. The charge $\mathrm{Q}(\xi)$ is independent of the choice of conformal completion even though the conformal completion is not unique. The choice $\bar{H}=\ell H r^{-1}$ provides a convenient conformal completion, smooth as $A \rightarrow 0$.

\footnotetext{
${ }^{2}$ Note that when Wick rotating Kerr $i a$ appears in the metric. However to check regularity at the horizon one must approach in a locally co-rotating frame: $\left(r_{+}^{2}+a^{2}\right) \varphi / K=-i a \tau / \alpha$. This results in a manifestly $\theta$-independent answer following the usual procedure. Alternately, using the zeroth law, the temperature is constant on the horizon, and hence can be evaluated at $\theta=0$ for simplicity.
} 
The angular momentum (3.1) is given by $\mathrm{Q}\left(-\partial_{\varphi}\right)$. As is well known [24], the angular potential of a rotating black hole in AdS is not simply the angular momentum at the horizon in Boyer-Lindquist coordinates, but must be corrected to allow for the relative rotation with the boundary. In general, the angular velocity of the "Zero-Angular-Momentum-Observer" any radius $r$ is

$$
\Omega_{r}=-\frac{g_{t \varphi}}{g_{\varphi \varphi}}=\frac{K a\left[h\left(r^{2}+a^{2}\right)-f r^{2}\right]}{\alpha\left[h\left(r^{2}+a^{2}\right)^{2}-f a^{2} r^{2} \sin ^{2} \theta\right]} .
$$

Setting $r=r_{+}$, this yields $\Omega_{H}$ in (3.1) on the horizon but is not constant on the boundary $H=0$. However, one can define

$$
\Omega_{\infty}=-\frac{a K\left(1-A^{2} \ell^{2} \Xi\right)}{\ell^{2} \Xi \alpha\left(1+a^{2} A^{2}\right)}
$$

by evaluating (3.9) at conformal infinity with $m=0$ (and $\cos \theta=1$ for the charged black hole). Similar to the Kerr-AdS case, it is the difference between the two, $\Omega=\Omega_{H}-\Omega_{\infty}$ that enters the first law of black hole thermodynamics.

$\boldsymbol{M}$. Moving to the thermodynamic mass, we used three methods to find the expression in (3.1). First, the conformal method with

$$
M=\mathrm{Q}\left(\partial_{t}+\Omega_{\infty} \partial_{\varphi}\right)
$$

yields the expression in (3.1).

We can check this result by computing the Gibbs free energy $\mathcal{G}=I / \beta=\mathcal{G}(T, \Omega, \Phi)$ from the action

$$
I=\frac{1}{16 \pi} \int_{M} d^{4} x \sqrt{g}\left[R+\frac{6}{\ell^{2}}-F^{2}\right]+\frac{1}{8 \pi} \int_{\partial M} d^{3} x \sqrt{h}\left[\mathcal{K}-\frac{2}{\ell}-\frac{\ell}{2} \mathcal{R}(h)\right],
$$

where $\mathcal{K}$ and $\mathcal{R}(h)$ are respectively the extrinsic curvature and Ricci scalar of the boundary. Computing this free energy is a bit lengthy, but straightforward, and yields

$$
\mathcal{G}=\frac{m\left(1-a^{2} A^{2}-2 A^{2} \ell^{2} \Xi\right)}{2 \alpha K\left(1+a^{2} A^{2}\right)}-\frac{r_{+}\left(a^{2}+r_{+}^{2}\right)}{2 \alpha K \ell^{2}\left(1-A^{2} r_{+}^{2}\right)^{2}}-\frac{e^{2} r_{+}}{2 K \alpha\left(a^{2}+r_{+}^{2}\right)},
$$

which satisfies $\mathcal{G}=M-\Omega J-T S-\Phi Q$ [33], with the thermodynamic variables in (3.1) as required.

The third cross-check is to use integration of the holographic stress tensor. In the fixed potential ensemble (without the $I_{Q}$ term), varying the action (3.12) (for generic variations that can include boundary diffeomorphisms [30]) gives

$$
\delta I=-\frac{1}{2} \int_{\partial M} \mathcal{T}_{a b} \delta h^{a b} \sqrt{-h} d^{3} x
$$

where

$$
8 \pi \mathcal{T}_{a b}=\ell \mathcal{G}_{a b}(h)-\frac{2}{\ell} h_{a b}-\mathcal{K}_{a b}+h_{a b} \mathcal{K} .
$$


In order to compute the quantities appearing in this expression, we require a systematic expansion of the metric near the boundary. This is typically done via a set of asymptotic Fefferman-Graham coordinates, in which the geometry takes a standard format

$$
d s^{2}=\frac{\ell^{2}}{z^{2}} d z^{2}+z^{-2}\left(\gamma_{a b}^{(0)}+z^{2} \gamma_{a b}^{(2)}+\ldots\right) d x^{a} d x^{b} .
$$

The transformation between the C-metric and FG coordinates is then written as an expansion in $z$, and expressions computed order by order. This process is straightforward but lengthy, and determines the $\gamma_{a b}^{(n)}$ in (3.16) above. Since $h_{a b}=\gamma_{a b}^{(0)} / z^{2}+\gamma_{a b}^{(2)}+\ldots$, it is now straightforward to compute $\mathcal{K}_{a b}$ in (3.15) leading to

$$
8 \pi \mathcal{T}_{a b}=\ell \mathcal{G}_{a b}+\frac{1}{\ell}\left[\gamma_{a b}^{(2)}+\frac{3 z}{2} \gamma_{a b}^{(3)}-\gamma_{a b}^{(0)} \gamma^{(0)^{c d}}\left(\gamma_{c d}^{(2)}+\frac{3 z}{2} \gamma_{c d}^{(3)}\right)\right]
$$

Inserting the computed values of $\gamma^{(n)}$, allows the computation of the expectation value of the energy momentum of the $\mathrm{CFT}_{3}$,

$$
\left\langle\mathcal{T}_{a b}\right\rangle=\lim _{z \rightarrow 0} \frac{\mathcal{T}_{a b}}{\ell z},
$$

yielding a relativistic fluid whose energy density can be integrated to give the mass. The details on this procedure are given in appendix A.

$\boldsymbol{\alpha}$. The crucial step in formulating the correct thermodynamics is to determine the normalization $\alpha$ in (3.2). For the uncharged and non-rotating black hole, this is straightforward to determine, as described in [21]: in the limit of vanishing black hole mass and string tensions, the spacetime becomes pure AdS in Rindler coordinates. The coordinate transformation between Rindler and global AdS then determines the rescaling of the time coordinate of the C-metric.

Alternatively, note that in this same limit, the boundary metric becomes (see eq. (A.6) in appendix A)

$$
d s_{(0)}^{2}=\omega^{2}\left[-\frac{d \tau^{2}}{\ell^{2}}+\frac{\alpha^{2} d x^{2}}{\left(1-x^{2}\right)\left(1-A^{2} \ell^{2}\left(1-x^{2}\right)\right)}+\frac{\alpha^{2}\left(1-x^{2}\right) d \varphi^{2}}{\left(1-A^{2} \ell^{2}\left(1-x^{2}\right)\right)}\right] .
$$

The latter two terms should correspond to a unit $S^{2}$, so we set

$$
\sin ^{2} \vartheta=\frac{\alpha^{2}\left(1-x^{2}\right)}{\left(1-A^{2} \ell^{2}\left(1-x^{2}\right)\right)} .
$$

Computing the $\vartheta$ component of the metric then gives

$$
\frac{\alpha^{2} \cos ^{2} \vartheta d \vartheta^{2}}{\alpha^{2}-\left(1-A^{2} \ell^{2}\right)+\left(1-A^{2} \ell^{2}\right) \cos ^{2} \vartheta}
$$

hence $\alpha^{2}=1-A^{2} \ell^{2}$.

For non-zero rotation similar approaches apply. Set $m=e=0$ in (2.1), and $K=1+a^{2} A^{2}-a^{2} / \ell^{2}$ so that there are no conical deficits present. We then rewrite 
the metric in a non-rotating frame by setting $\varphi=\tilde{\phi}+\Omega_{\infty} t$, thus eliminating the $g_{t \tilde{\phi}}$ component of the metric:

$$
d s^{2}=\frac{1}{H^{2}}\left(-\frac{\tilde{f} \tilde{h} d t^{2}}{\alpha^{2} \tilde{\Xi}}+\frac{r^{2} \Sigma d r^{2}}{\left(r^{2}+a^{2}\right) \tilde{f}}+\frac{r^{2} \Sigma d \theta^{2}}{\tilde{h}}+\frac{\sin ^{2} \theta\left(r^{2}+a^{2}\right) d \tilde{\phi}^{2}}{\tilde{\Xi}}\right),
$$

where

$$
\tilde{f}=1-A^{2} r^{2}+\frac{r^{2}}{\ell^{2}}, \quad \tilde{h}=1+\left(a^{2} A^{2}-\frac{a^{2}}{\ell^{2}}\right) \cos ^{2} \theta, \quad \tilde{\Xi}=K=1+a^{2} A^{2}-\frac{a^{2}}{\ell^{2}} .
$$

This is to be compared with the AdS metric:

$$
d s^{2}=-\left(1+\frac{R^{2}}{\ell^{2}}\right) d t^{2}+\frac{d R^{2}}{1+\frac{R^{2}}{\ell^{2}}}+R^{2}\left(d \Theta^{2}+\sin ^{2} \Theta d \tilde{\phi}^{2}\right) .
$$

Equality of the $g_{t t}$ and $g_{\tilde{\phi} \tilde{\phi}}$ components in each case determines $R(r, \theta)$ and $\Theta(r, \theta)$ :

$$
R \sin \Theta=\frac{\sin \theta \sqrt{r^{2}+a^{2}}}{H \sqrt{\tilde{\Xi}}}, \quad R=\ell \sqrt{\frac{\tilde{f} \tilde{h}}{H^{2} \alpha^{2} \tilde{\Xi}}-1} .
$$

Then imposing that the remaining metric components agree yields $\alpha=\sqrt{1-A^{2} \ell^{2}}$, as in the non-rotating case.

$\boldsymbol{V}, \boldsymbol{\lambda}_{ \pm}$. The remaining thermodynamic variables are the pressure, string tensions, and their dual potentials. The definition of the charges $P, \mu_{ \pm}$are fixed in terms of the physical quantities: the cosmological constant determines $P=3 / 8 \pi \ell^{2}$, and the tensions are fixed by the conical deficits (2.7). The conjugate potentials, the thermodynamic volume and lengths are then determined from the first law (1.1). This is computed by first looking at how the location of the black hole event horizon changes as we vary the metric parameters

$$
\frac{\partial f}{\partial r_{+}} \delta r_{+}+\frac{\partial f}{\partial m} \delta m+\frac{\partial f}{\partial e} \delta e+\frac{\partial f}{\partial a} \delta a+\frac{\partial f}{\partial \ell} \delta \ell+\frac{\partial f}{\partial A} \delta A+\frac{\partial f}{\partial K} \delta K=0 .
$$

Next, $\delta r_{+}$(conveniently multiplied by $f_{+}^{\prime} \propto T$ ) can be rewritten in terms of $\delta S, \delta a, \delta A$, and $\delta K$. This now gives $T \delta S$ in terms of $\delta m, \delta e, \delta a \delta \ell, \delta A$, and $\delta K$. We then use the expressions for $M, Q, J, P$ in (3.1) and $\mu_{ \pm}$in (2.7) to transform this into the form of the first law. This procedure results in the expressions for $V$ and $\lambda_{ \pm}$in (3.1).

\section{On thermodynamics of the asymptotically flat C-metric}

It is also interesting to consider what happens if there is an acceleration horizon. For simplicity, we concentrate on the asymptotically flat limit of the accelerated black holes, ${ }^{3}$ obtained by setting $\Lambda=0$ in (2.1). It describes a pair of accelerating black holes separated

\footnotetext{
${ }^{3}$ Alternatively, one could consider the 'fast accelerating' AdS black holes. Such spacetimes have both accelerated and cosmological horizons extending all the way to conformal infinity and the standard holographic and conformal methods are not so readily applicable for their study.
} 
by an acceleration horizon, and we note that its conformal structure is analogous to de Sitter spacetime, but with future/past infinity being null at the poles $\theta=0, \pi$ and otherwise spacelike [34].

Given this clear physical distinction, finding the thermodynamic variables is not a matter of taking the limit $\ell \rightarrow \infty$ for each quantity in (3.1); indeed such a limit does not exist. Rather we concentrate on an observer in the static patch of (2.1), between one black hole and its acceleration horizon. Despite the fact that charged rotating black holes emit gravitational and electromagnetic radiation at infinity [37, 38], observers using the metric (2.1) should be able to undertake standard thermodynamic investigations, since the radiation escapes into a region inaccessible to this observer [39]. Of course, due to the presence of two horizons such a system is in general out of equilibrium. We expect that similar to the de Sitter case, one may study the thermodynamics of each horizon separately [40].

Leaving the thermodynamics of the accelerated horizon for future study (see $[35,36]$ ), with this in mind we find

$$
\begin{aligned}
M & =\frac{m\left(1-a^{2} A^{2}\right)}{K \alpha\left(1+a^{2} A^{2}\right)}, \quad T=\frac{f_{+}^{\prime} r_{+}^{2}}{4 \pi \alpha\left(r_{+}^{2}+a^{2}\right)}, \quad S=\frac{\pi\left(r_{+}^{2}+a^{2}\right)}{K\left(1-A^{2} r_{+}^{2}\right)}, \\
\alpha & =\frac{\sqrt{\left(1-a^{2} A^{2}\right) \Xi}}{1+a^{2} A^{2}}, \quad Q=\frac{e}{K}, \quad \Phi=\frac{e r_{+}}{\left(a^{2}+r_{+}^{2}\right) \alpha}, \\
J & =\frac{m a}{K^{2}}, \quad \Omega=\Omega_{H}-\Omega_{\infty}=\frac{a K}{\alpha\left(r_{+}^{2}+a^{2}\right)}-\frac{a A^{2} K}{\alpha\left(1+a^{2} A^{2}\right)}, \\
\lambda_{ \pm} & =\frac{r_{+}}{\alpha\left(1 \pm A r_{+}\right)}-\frac{M K}{\Xi} \mp \frac{a^{2} A}{\alpha\left(1+a^{2} A^{2}\right)},
\end{aligned}
$$

form a consistent set of thermodynamic parameters for the $\Lambda=0$ charged accelerating black hole, satisfying (with $P=0$ ) both the first law (1.1) and the Smarr relation (1.2) at the black hole horizon, where $\Xi=\left(1+a^{2} A^{2}+e^{2} A^{2}\right)$. The quantity $M$ was computed via a Komar-like integral over $\theta$ and $\varphi$ at conformal infinity, using a Killing vector $\partial_{t}$ in a rotation subtracted frame $\varphi \rightarrow \varphi+\Omega_{\infty} t$. Note that it is not possible to compute $V$ in this case, as no smooth limit exists for $V$ as $P \rightarrow 0$, similar to what happens for black holes in $d=7$ gauged supergravity [41].

A much greater measure of caution should be taken before the formulae in (4.1) are accepted as thermodynamic quantities. In computing the mass the Killing vector $\partial_{t}$ was used; this is a boost rotation Killing vector rather than the static vector at infinity. Moreover, spatial infinity is not well described in the static coordinates - it only corresponds to $\theta=\pi / 2$ in these coordinates, calling into question the validity of the Komar integration. Third, the above quantities satisfy the first law only provided this Killing vector is normalized by $\alpha$ above. Whereas there is a good reason to do this in the asymptotically AdS case studied in the main text, such a normalization remains a question in the asymptotically flat case. Finally, the physical status of the thermodynamic relations is not clear given the presence of radiation [37, 38]; indeed even in the slowly accelerating AdS case radiation patterns have been computed [37]. 


\section{Discussion and summary}

To summarize, we have formulated the consistent thermodynamics of slowly accelerating AdS black holes with charge and rotation. The crucial aspects of our result are the computation of the thermodynamic mass and the normalization of the timelike Killing vector. We computed the mass directly via the conformal method, and backed up this result by checking it against the free energy from a calculation of the action and (for zero rotation) by calculating the holographic energy momentum tensor and its associated energy. The mutual agreement of these methods is not obvious, as the Dirichlet boundary conditions no longer apply to the case in question [31]. The normalization of the timelike Killing vector was cross-checked by finding explicit coordinate transformations and (for zero rotation) by requiring the variation of the action (including boundary counterterms) to vanish.

The key insight in our approach is to consider the extended first law (1.1) with the additional terms that allow us to vary the tension. This is in direct contrast to the approach presented in [19] (see also [32]) where one tension is fixed (at zero) and all remaining parameters are permitted to vary. This is perplexing, as a first law without varying tension then has reduced cohomogeneity, i.e. fewer thermodynamic parameters than the number of parameters being varied in the geometry. Given this mismatch of parameter space, we suspect a lack of uniqueness, or a hidden constraint.

We have also proposed a set of thermodynamic parameters for the $\Lambda=0$ charged and accelerating black hole spacetime. This spacetime has an acceleration horizon, but (as is the case with other black holes) the first law and Smarr formula hold at the black hole horizon.

Our results pave the way for a proper analysis of the phase behaviour of accelerating black holes and raise a number of interesting questions for future study. Standard thermodynamic analysis seems to be possible for observers using the metric (2.1) (e.g. [43]) despite the fact that charged rotating black holes emit gravitational and electromagnetic radiation at infinity [37, 38], since the coordinates from which we obtained our thermodynamic parameters are analogous to those of an observer comoving with an accelerating charged object, who sees no radiation because it escapes into a region inaccessible to this observer [39]. It is an interesting future project to obtain a deeper understanding of this relationship. Similar remarks apply to the $\Lambda \geq 0$ cases as well as the fast accelerating AdS case, though the loss of thermodynamic equilibrium (duel to the presence of additional horizons) introduces new complications. The quantities in (4.1) were with regard to one accelerated black hole in the static patch. It remains an interesting question whether one should not rather treat the system of two black holes as a whole, reminiscent of the mass dipole, and consider the associated boost mass [44].

It is also of interest to obtain a better understanding of these black holes from a holographic perspective. While we have checked our expression for the mass using this approach, a full understanding of the dual fluid interpretation for the charged and rotating case remains to be found. It likewise would be preferable to obtain a clear understanding of the conserved mass for the $\Lambda \geq 0$ cases, since standard holographic methods do not directly apply. It would also be interesting to see how changes in the boundary metric affect the shape of these AdS black holes [45]. 


\section{Acknowledgments}

We would like to thank Michael Appels, Pavel Krtouš, Rob Myers, and Kostas Skenderis for useful discussions. This work was supported in part by the Alexander von Humboldt foundation (AA), a Chilean FONDECYT [Grant No. 1181047 (AA), 1170279 (AA), 1161418 (AA)], CONYCT-RCUK [Newton-Picarte Grants DPI20140053 (AA) and DPI20140115 (AA)], by the STFC [consolidated grant ST/P000371/1 (RG)], the Natural Sciences and Engineering Research Council of Canada (DK and RBM), and by the Perimeter Institute (FG, DK and RG). Research at Perimeter Institute is supported by the Government of Canada through the Department of Innovation, Science and Economic Development and by the Province of Ontario through the Ministry of Research and Innovation. RG would also like to thank the Simons Foundation for support and the Aspen Center for Physics for hospitality while this work was being completed.

\section{A Holographic stress tensor}

In this appendix we provide further detail, and gather the formulae for the holographic stress tensor and boundary metric variations, concentrating mainly on the slowly accelerating charged (non-rotating) AdS C-metric, as the expressions once rotation is included are extremely cumbersome.

The first step is to write a coordinate transformation between the C-metric coordinates in (2.1) and the FG coordinates of (3.16) in a series expansion ${ }^{4}$ as $z \rightarrow 0$ :

$$
\frac{1}{r}=-A x-\sum_{n=1}^{4} F_{n}(x) z^{n}, \quad \cos \theta=x+\sum_{n=1}^{4} G_{n}(x) z^{n} .
$$

One inserts this expansion (A.1) into (2.1) and inspects the terms order by order in $z$. The functions $F_{n}$ and $G_{n}$ are then found by requiring that $g_{z z} \equiv \ell^{2} / z^{2}$, and $g_{z a} \equiv 0$ at each order in $z$; this determines all but the function $F_{1}$, which appears as an overall conformal factor as expected. The $\gamma_{a b}^{(n)}$ are also determined by these expressions.

To illustrate the process, note that

$$
g_{z x}=0 \quad \Rightarrow \quad \frac{1}{h} \frac{\partial \theta}{\partial z} \frac{\partial \theta}{\partial x}=-\frac{r^{2}}{f} \frac{\partial r^{-1}}{\partial z} \frac{\partial r^{-1}}{\partial x}
$$

which implies

$$
\begin{aligned}
\frac{1}{\ell^{2}}\left(\sum n G_{n} z^{n-1}\right) & \left(A+\sum G_{n}^{\prime}(x) z^{n}\right) \Upsilon_{a}^{2}\left[x+\sum \frac{F_{n}}{A} z^{n}\right] \\
= & -\left(\sum n F_{n} z^{n-1}\right)\left(A+\sum F_{n}^{\prime}(x) z^{n}\right) X\left[x+\sum G_{n} z^{n}\right],
\end{aligned}
$$

where

$$
\begin{aligned}
X[\xi] & =\left(1-\xi^{2}\right)\left(1+2 m A \xi+\left(e^{2}+a^{2}\right) A^{2} \xi^{2}-a^{2} \xi^{2} / \ell^{2}\right), \\
\Upsilon_{a}[\xi] & =\sqrt{1+a^{2} A^{2} \xi^{4}-A^{2} \ell^{2} X(\xi)},
\end{aligned}
$$

\footnotetext{
${ }^{4}$ Note that the $r$-coordinate in (2.1) only covers half of the boundary, since $r \rightarrow \infty$ at $\theta=\pi / 2$, therefore we use $y=1 / r$ as an alternate radial coordinate.
} 
have been written to keep notation concise. A systematic expansion, order by order, then determines the $G_{n}$ in terms of the $F_{n}$. For example, at leading order, $\mathcal{O}\left(z^{-2}\right)$, gives

$$
G_{1}(x)=-\frac{A \ell^{2} F_{1}(x) X(x)}{\Upsilon_{a}^{2}(x)} .
$$

This then is sufficient to ensure $g_{z z}=\ell^{2} / z^{2}$ to this order, and gives the boundary metric as:

$$
d s_{(0)}^{2}=\frac{\Upsilon_{a}^{4}}{\Sigma_{F G}^{3} F_{1}^{2}}\left(X\left[a A^{2} x^{2} \frac{d t}{\alpha}-\left(1+a^{2} A^{2} x^{2}\right) \frac{d \varphi}{K}\right]^{2}-\frac{\Upsilon_{a}^{2}}{\ell^{2}}\left[\frac{d t}{\alpha}-a\left(1-x^{2}\right) \frac{d \varphi}{K}\right]^{2}\right)+\frac{\Upsilon_{a}^{2}}{F_{1}^{2} X} d x^{2},
$$

where, again for conciseness, we write

$$
\Sigma_{F G}=\left(1+a^{2} A^{2} x^{4}\right),
$$

and the arguments of all the functions are understood now as being ' $x$ '. Note that the transformation (A.1) is valid in general only when $\Upsilon_{a}^{2}>0$, which precisely translates into the condition that acceleration horizons are absent, i.e. we are working with the slowly accelerating C-metric.

To proceed further, we must now Taylor expand the functions $X$ and $\Upsilon_{a}^{2}$ in (A.3), and the expressions rapidly become rather lengthy for $a \neq 0$. Setting $a=0$, which sets $\Sigma_{F G}=1, \Upsilon_{a}=\Upsilon=\sqrt{1-A^{2} \ell^{2} X}$, and further writing $F_{1}(x)=-\Upsilon^{3} / \alpha \omega(x)$ in order to elucidate the conformal degree of freedom in the boundary metric, $\omega$, allows a more tractable computation of the $\gamma^{(n)}$, and hence the stress tensor. In coordinates $(t, x, \varphi)$, the expectation value of the energy momentum of the $\mathrm{CFT}_{3}$ reads

$$
\left\langle\mathcal{T}_{b}^{a}\right\rangle=\lim _{z \rightarrow 0} \frac{1}{\ell z} \mathcal{T}_{b}^{a}=-\frac{X^{\prime \prime \prime}(x) \Upsilon^{3}}{96 \pi A \alpha^{3} \omega^{3}} \operatorname{diag}\left[-\left(2-3 A^{2} \ell^{2} X\right), 1,\left(1-3 A^{2} \ell^{2} X\right)\right] .
$$

This can be interpreted as an energy momentum tensor of a thermal perfect fluid with no dissipation terms plus a non-hydrodynamic correction [21, 42]. With respect to the static observer at infinity, $U=\omega^{-1} \partial_{\tau}$, this yields the following energy density:

$$
\rho_{E}=\frac{\left(m+2 e^{2} A x\right)}{8 \pi \ell^{2} \alpha^{3} \omega^{3}} \Upsilon^{3}\left(2-3 A^{2} \ell^{2} X\right),
$$

which upon integration, yields the holographic mass

$$
M=\int \rho_{E} \ell^{3} \sqrt{-\gamma^{(0)}} d x d \varphi=\frac{m\left(1-A^{2} \ell^{2}-A^{4} e^{2} \ell^{2}\right)}{K \alpha},
$$

in agreement with (3.1). Finally, we find the variation of the boundary metric with respect to the parameters,

$$
\delta \gamma^{a b}=\frac{\partial \gamma^{a b}}{\partial K} \delta K+\frac{\partial \gamma^{a b}}{\partial A} \delta A+\frac{\partial \gamma^{a b}}{\partial m} \delta m+\frac{\partial \gamma^{a b}}{\partial e} \delta e,
$$

keeping $\ell$ and $\mu$ 's constant, and calculate

$$
\delta I=\int_{\partial M} \sqrt{-\gamma} \tau_{a b} \delta \gamma^{a b} d^{3} x .
$$

Imposing that the variation vanishes we find that the unknown parameter $\alpha$ must be

$$
\alpha=\sqrt{\left(1+A^{2} e^{2}\right)\left(1-A^{2} \ell^{2}-A^{4} \ell^{2} e^{2}\right)},
$$

which agrees with (3.2) in the case $a=0$. 
Open Access. This article is distributed under the terms of the Creative Commons Attribution License (CC-BY 4.0), which permits any use, distribution and reproduction in any medium, provided the original author(s) and source are credited.

\section{References}

[1] J.D. Bekenstein, Black holes and entropy, Phys. Rev. D 7 (1973) 2333 [InSPIRE].

[2] J.D. Bekenstein, Generalized second law of thermodynamics in black hole physics, Phys. Rev. D 9 (1974) 3292 [INSPIRE].

[3] S.W. Hawking, Particle Creation by Black Holes, Commun. Math. Phys. 43 (1975) 199 [Erratum ibid. 46 (1976) 206] [INSPIRE].

[4] D. Kubizňák, R.B. Mann and M. Teo, Black hole chemistry: thermodynamics with Lambda, Class. Quant. Grav. 34 (2017) 063001 [arXiv:1608.06147] [INSPIRE].

[5] W. Kinnersley and M. Walker, Uniformly accelerating charged mass in general relativity, Phys. Rev. D 2 (1970) 1359 [InSPIRE].

[6] J.F. Plebański and M. Demiański, Rotating, charged and uniformly accelerating mass in general relativity, Annals Phys. 98 (1976) 98 [INSPIRE].

[7] O.J.C. Dias and J.P.S. Lemos, Pair of accelerated black holes in anti-de Sitter background: AdS C metric, Phys. Rev. D 67 (2003) 064001 [hep-th/0210065] [INSPIRE].

[8] J.B. Griffiths and J. Podolsky, A New look at the Plebański-Demiański family of solutions, Int. J. Mod. Phys. D 15 (2006) 335 [gr-qc/0511091] [InSPIRE].

[9] R. Gregory and M. Hindmarsh, Smooth metrics for snapping strings, Phys. Rev. D 52 (1995) 5598 [gr-qc/9506054] [INSPIRE].

[10] F. Dowker, J.P. Gauntlett, D.A. Kastor and J.H. Traschen, Pair creation of dilaton black holes, Phys. Rev. D 49 (1994) 2909 [hep-th/9309075] [InSPIRE].

[11] S.W. Hawking, G.T. Horowitz and S.F. Ross, Entropy, Area and black hole pairs, Phys. Rev. D 51 (1995) 4302 [gr-qc/9409013] [INSPIRE].

[12] R. Emparan, Pair creation of black holes joined by cosmic strings, Phys. Rev. Lett. 75 (1995) 3386 [gr-qc/9506025] [inSPIRE].

[13] D.M. Eardley, G.T. Horowitz, D.A. Kastor and J.H. Traschen, Breaking cosmic strings without monopoles, Phys. Rev. Lett. 75 (1995) 3390 [gr-qc/9506041] [INSPIRE].

[14] R.B. Mann and S.F. Ross, Cosmological production of charged black hole pairs, Phys. Rev. D 52 (1995) 2254 [gr-qc/9504015] [INSPIRE].

[15] I.S. Booth and R.B. Mann, Complex instantons and charged rotating black hole pair creation, Phys. Rev. Lett. 81 (1998) 5052 [gr-qc/9806015] [inSPIRE].

[16] M. Appels, R. Gregory and D. Kubizňák, Thermodynamics of Accelerating Black Holes, Phys. Rev. Lett. 117 (2016) 131303 [arXiv:1604.08812] [INSPIRE].

[17] M. Appels, R. Gregory and D. Kubizňák, Black Hole Thermodynamics with Conical Defects, JHEP 05 (2017) 116 [arXiv: 1702.00490] [INSPIRE].

[18] R. Gregory, Accelerating Black Holes, J. Phys. Conf. Ser. 942 (2017) 012002 [arXiv: 1712.04992] [INSPIRE]. 
[19] M. Astorino, CFT Duals for Accelerating Black Holes, Phys. Lett. B 760 (2016) 393 [arXiv: 1605.06131] [INSPIRE].

[20] M. Astorino, Thermodynamics of Regular Accelerating Black Holes, Phys. Rev. D 95 (2017) 064007 [arXiv: 1612.04387] [INSPIRE].

[21] A. Anabalón, M. Appels, R. Gregory, D. Kubizňák, R.B. Mann and A. Ovgün, Holographic Thermodynamics of Accelerating Black Holes, Phys. Rev. D 98 (2018) 104038 [arXiv: 1805.02687] [INSPIRE].

[22] J. Podolsky, Accelerating black holes in anti-de Sitter universe, Czech. J. Phys. 52 (2002) 1 [gr-qc/0202033] [INSPIRE].

[23] L. Smarr, Mass formula for Kerr black holes, Phys. Rev. Lett. 30 (1973) 71 [Erratum ibid. 30 (1973) 521] [INSPIRE].

[24] G.W. Gibbons, M.J. Perry and C.N. Pope, The First law of thermodynamics for Kerr-anti-de Sitter black holes, Class. Quant. Grav. 22 (2005) 1503 [hep-th/0408217] [INSPIRE].

[25] V.E. Hubeny, D. Marolf and M. Rangamani, Black funnels and droplets from the AdS C-metrics, Class. Quant. Grav. 27 (2010) 025001 [arXiv:0909.0005] [INSPIRE].

[26] C. Herdeiro, B. Kleihaus, J. Kunz and E. Radu, On the Bekenstein-Hawking area law for black objects with conical singularities, Phys. Rev. D 81 (2010) 064013 [arXiv:0912.3386] [INSPIRE].

[27] S.W. Hawking and S.F. Ross, Duality between electric and magnetic black holes, Phys. Rev. D 52 (1995) 5865 [hep-th/9504019] [INSPIRE].

[28] A. Ashtekar and S. Das, Asymptotically Anti-de Sitter space-times: Conserved quantities, Class. Quant. Grav. 17 (2000) L17 [hep-th/9911230] [INSPIRE].

[29] S. Das and R.B. Mann, Conserved quantities in Kerr-anti-de Sitter space-times in various dimensions, JHEP 08 (2000) 033 [hep-th/0008028] [INSPIRE].

[30] I. Papadimitriou and K. Skenderis, Thermodynamics of asymptotically locally AdS spacetimes, JHEP 08 (2005) 004 [hep-th/0505190] [INSPIRE].

[31] S. Hollands, A. Ishibashi and D. Marolf, Comparison between various notions of conserved charges in asymptotically AdS-spacetimes, Class. Quant. Grav. 22 (2005) 2881 [hep-th/0503045] [INSPIRE].

[32] M. Astorino, G. Compère, R. Oliveri and N. Vandevoorde, Mass of Kerr-Newman black holes in an external magnetic field, Phys. Rev. D 94 (2016) 024019 [arXiv:1602.08110] [InSPIRE].

[33] M.M. Caldarelli, G. Cognola and D. Klemm, Thermodynamics of Kerr-Newman-AdS black holes and conformal field theories, Class. Quant. Grav. 17 (2000) 399 [hep-th/9908022] [INSPIRE].

[34] J.B. Griffiths, P. Krtouš and J. Podolsky, Interpreting the C-metric, Class. Quant. Grav. 23 (2006) 6745 [gr-qc/0609056] [InSPIRE].

[35] E. Bianchi and A. Satz, Mechanical laws of the Rindler horizon, Phys. Rev. D 87 (2013) 124031 [arXiv: 1305.4986 ] [INSPIRE].

[36] T. De Lorenzo and A. Perez, Light Cone Thermodynamics, Phys. Rev. D 97 (2018) 044052 [arXiv:1707.00479] [INSPIRE]. 
[37] J. Podolsky, M. Ortaggio and P. Krtouš, Radiation from accelerated black holes in an anti-de Sitter universe, Phys. Rev. D 68 (2003) 124004 [gr-qc/0307108] [InSPIRE].

[38] J. Podolsky and H. Kadlecova, Radiation generated by accelerating and rotating charged black holes in (anti-)de Sitter space, Class. Quant. Grav. 26 (2009) 105007 [arXiv:0903.3577] [INSPIRE].

[39] D.G. Boulware, Radiation From a Uniformly Accelerated Charge, Annals Phys. 124 (1980) 169 [INSPIRE].

[40] B.P. Dolan, D. Kastor, D. Kubiznák, R.B. Mann and J. Traschen, Thermodynamic Volumes and Isoperimetric Inequalities for de Sitter Black Holes, Phys. Rev. D 87 (2013) 104017 [arXiv:1301.5926] [INSPIRE].

[41] M. Cvetič, G.W. Gibbons, D. Kubizňák and C.N. Pope, Black Hole Enthalpy and an Entropy Inequality for the Thermodynamic Volume, Phys. Rev. D 84 (2011) 024037 [arXiv: 1012.2888] [INSPIRE].

[42] G. Bernardi de Freitas and H.S. Reall, Algebraically special solutions in AdS/CFT, JHEP 06 (2014) 148 [arXiv: 1403.3537] [INSPIRE].

[43] J. Zhang, Y. Li and H. Yu, Thermodynamics of charged accelerating AdS black holes and holographic heat engines, JHEP 02 (2019) 144 [arXiv: 1808.10299] [INSPIRE].

[44] K. Dutta, S. Ray and J. Traschen, Boost mass and the mechanics of accelerated black holes, Class. Quant. Grav. 23 (2006) 335 [hep-th/0508041] [INSPIRE].

[45] G.T. Horowitz, J.E. Santos and C. Toldo, Deforming black holes in AdS, JHEP 11 (2018) 146 [arXiv: 1809.04081] [INSPIRE]. 